\title{
Cultura escolar, prácticas de enseñanza $y$ resiliencia en alumnos y profesores de contextos sociales vulnerables*
}

\author{
School culture, practices and resilience in education, \\ students and teachers of vulnerable social contexts
}

Recibido: junio 15 de 2010 | Revisado: julio 23 de 2010 | Aceptado: septiembre 9 de 2010

\author{
Marco Antonio Villalta Paucar ** \\ Universidad de Santiago de Chile, Santiago, Chile \\ EugENiO SAAVEDRA GUAJARDO**** \\ Universidad Católica del Maule, Chile
}

SICI: 2011-2777(201203)11:1<67:CEPERA>2.0.CO;2-9

Para citar este artículo: Villalta, M. A. \& Saavedra, E. (2012). Cultura escolar, prácticas de enseñanza y resiliencia en alumnos y profesores de contextos sociales vulnerables. Universitas Psychologica, 11(1), $67-78$.

* El trabajo forma parte del Proyecto FONDECYT №1095049.

** Escuela de Psicología. Av. Ecuador 3650, Estación Central, Santiago, Chile. Email: marco.villalta@ usach.cl. ResearcherID: Villalta, M. A., B-95552012.

***: Escuela de Psicología. Av. San Miguel 3605, Talca. E-mail: esaavedr@ucm.cl
RES UMEN

Se analiza en qué medida aspectos como la percepción de la cultura institucional, de las prácticas de enseñanza en sala de clase y la resiliencia de alumnos y profesores, están asociados a logros educativos, en instituciones ubicadas en sectores sociales vulnerables. Se presenta un estudio cuantitativo de tipo descriptivo correlacional, con 502 alumnos y 39 profesores de dos establecimientos educativos con adecuados logros de aprendizaje, ubicados en zonas sociales vulnerables. Se mide percepción de: 1) Cultura Institucional, 2) Prácticas cotidianas de enseñanza y 3) Resiliencia. En los alumnos se encuentra alta correlación entre percepciones de cultura institucional y el trabajo cotidiano de enseñanza. Dichas percepciones tienen correlación moderada con la Resiliencia. En los profesores se encuentra correlación moderada entre sus percepciones de cultura y prácticas de enseñanza, y nula correlación de ambas con la resiliencia. Se profundiza y discuten estos resultados.

Palabras clave autores:

Vulnerabilidad, resiliencia, prácticas de enseñanza, cultura escolar.

Palabras clave descriptores:

Jóvenes, docentes, colegio, educación.

\section{A B S T R A C T}

In this article is examined the level that issues such as perception of institutional culture, teaching practices inside the classroom and students and teachers' resilience are associated with vulnerable social institutions educational achievement. We present a quantitative study of descriptive correlational, with 502 students and 39 teachers at two educational institutions with acceptable learning achievements which are located in vulnerable social areas. Perception is measured by: 1) Institutional culture, 2) daily Teaching practices, and 3) Resilience. In students perceptions of institutional culture and the daily work of teaching are high correlated. These perceptions have moderate correlation with resilience. On the other hand, teachers' of school culture and practices are medium correlated, and there is no correlation of both with resilience. These results are discussed and deepened.

Key words authors:

Vulnerability, resilience, teaching practices, school culture.

Key words plus:

Young people, teachers, school, education. 


\section{Introducción}

Diversos estudios constatan que la situación social de pobreza socioeconómica es un factor de riesgo psicosocial, donde son especialmente vulnerables los niños y adolescentes. Específicamente, en el ámbito de la educación formal chilena, se encuentra que en los sectores sociales vulnerables está la mayor cantidad de establecimientos educativos con bajos logros de aprendizaje. Sin embargo, no existe una relación causal entre dichas situaciones, pues hay un importante papel de la experiencia escolar que influye de modo determinante en lo que los alumnos pueden aprender (Fondo de Naciones Unidas para la Infancia [Unicef], 2004; Murillo \& Román, 2009; Villalta, 2009). Por tanto, es relevante conocer cuáles son las percepciones de alumnos y profesores sobre la organización escolar, las prácticas de enseñanza en el aula y las propias capacidades para enfrentar la adversidad, que pueden ayudar a comprender el buen logro educativo en estos contextos sociales.

El objetivo del presente estudio es describir la percepción de la cultura escolar, prácticas de enseñanza en sala de clase y resiliencia de alumnos y profesores pertenecientes a establecimientos educativos de sectores sociales vulnerables, con alto logro de aprendizaje, en comparación con sus pares institucionales y sociales.

\section{Marco de referencia teórico}

\section{El concepto de resiliencia y su medición}

En el área de las Ciencias Sociales y la Educación, se distinguen definiciones de resiliencia que comparten aspectos como: a) reconocer que es una capacidad humana más o menos universal (Grotberg, 1995; Rutter, 1993); b) está presente la idea de adversidad, estrés o contexto negativo (Grotberg, 1999; Manciaux, 2005); c) el sujeto es quien enfrenta las adversidades (Goodyer, 1995; Kreisler, 1996; Masten, 1990) y d) se obtiene como resultado una adaptación positiva (Goodyer, 1995; Lutha \& Cicchetti, 2000; Vanistendael, 2002).

La resiliencia más allá de ser una condición permanente y dependiente de factores externos a la persona, es una construcción propia y que varía según los contextos y los momentos en que ocurre (Saavedra, 2003). En último término, el considerarse resiliente o no será un evaluación interna que hace el sujeto frente a su vida. La medición de la resiliencia debe integrar una concepción dinámica que resalte el papel del sujeto en esta evaluación, sin desconocer que existen aspectos estructurales de la persona que sirven de base para la construcción de la conducta resiliente (Saavedra \& Villalta, 2008a).

La articulación entre distintos enfoques actuales de la resiliencia (Grotberg, 1995; Guidano, 1994; Saavedra, 2003) permite generar un camino de respuesta, que considere diferentes dimensiones internas de la persona, como lo hacen los modelos de Grotberg (1995) y de Saavedra (2003), que recogen el sentido histórico de la respuesta ante el obstáculo.

En esta dirección, la escala SV-RES (Saavedra \& Villalta, 2008a) considera modalidades de interacción del sujeto: a) consigo mismo, b) con los otros y c) con sus posibilidades, en relación con acciones para aprender y transformar proactivamente sus juicios, sobre 1) la capacidad de generar metas orientadoras de la acción, 2) la capacidad para resolver una situación problemática, 3) la definición de sí mismo y 4) la definición de la historia que la constituye como

TABLA 1

Dimensiones de la resiliencia en SV-RES (Saavedra \& Villalta, 2008a)

\begin{tabular}{lllll}
\hline Competencias interaccionales & \multicolumn{4}{c}{ Niveles de estructuración de la conciencia de Saavedra (2003) } \\
\cline { 2 - 5 } de Grotberg (1995) & Condiciones de base & Visión de sí mismo & Visión del problema & Respuesta resiliente \\
\hline Yo soy, yo estoy & 1: Identidad. & 2: Autonomía & 3: Satisfacción & 4: Pragmatismo \\
Yo tengo... & 5: Vínculos & 6: Redes & 7: Modelos & 8: Metas \\
Yo puedo & 9: Afectividad & 10: Autoeficacia & 11: Aprendizaje & 12: Generatividad \\
\hline
\end{tabular}

Fuente: elaboración propia. 
tal. El articular estos modelos permite distinguir 12 dimensiones que se presentan en la siguiente tabla.

Investigaciones recientes con la escala SV-RES en jóvenes y adultos, indican que no hay diferencias significativas al comparar jóvenes de diferentes niveles socioeconómicos; que no existe una relación directa entre el nivel de resiliencia y el rendimiento escolar (Villalta, 2009); que no hay relación entre el nivel de resiliencia y el género de los jóvenes ( $\mathrm{Sa}$ avedra \& Villalta, 2008a, 2008b); que no hay relación entre el nivel de resiliencia y el tramo de edad de los sujetos (Saavedra \& Villalta, 2008b) y que se observan diferencias significativas al comparar sujetos que provienen de familias biparentales nucleares y jóvenes internos en hogares de protección (Saavedra \& Yanquez, 2008).

\section{Vulnerabilidad social y educación}

El uso común del término vulnerabilidad se asocia a riesgo, fragilidad, indefensión o potencial daño. En este sentido, el Diccionario de la Real Academia de la Lengua Española afirma que la vulnerabilidad se refiere a 'la probabilidad de ser dañado o herido'. Estas acepciones, pertinentes al ámbito de la salud física o mental, llevadas al campo social han sido asociadas a la pobreza y, en términos más genéricos, a las situaciones de exclusión, que son los efectos materiales del riesgo. En tal sentido, la vulnerabilidad social comprende tanto la exposición al riesgo -probabilidad de caer en situación de pobreza y exclusión- como la capacidad para enfrentarlo, sea con recursos internos o apoyados en factores externos (Chambers, 1989).

Sin embargo, la relación de equivalencia entre exposición al riesgo y capacidad para enfrentarlo puede entenderse como una efectiva posibilidad de adaptarse al riesgo; pero esta simple adaptación implica un rol pasivo del sujeto, que equivale a la resignación y en consecuencia una forma encubierta de exclusión. Una adaptación activa, en cambio, implica una respuesta que conlleva reestructuraciones internas de la persona y de los grupos, así como revalorizaciones de los riesgos (Moser, 1993). En tal sentido, la vulnerabilidad social resulta de una sumatoria que contiene una condición social de riesgo a la pobreza y exclusión, más la incapacidad de los sujetos para enfrentarlos y la inhabilidad de adaptarse activamente a la situación, afectando en lo inmediato o en el futuro su bienestar en un contexto sociohistórico y cultural determinado (Perona \& Rocchi, 2001).

En el ámbito sociodemográfico, el concepto de vulnerabilidad se relaciona con grupos socialmente en riesgo, cuya identificación responde a diferentes criterios: la existencia de elementos contextuales que los hace más propensos a enfrentar situaciones adversas para su desarrollo, el ejercicio de conductas que los exponen a peligros y la presencia de atributos básicos que originan riesgos (edad, sexo, etnia).

Una perspectiva del riesgo social contemporáneo la ofrece Esping-Andersen (2000), que distingue cuatro tipos de riesgos: 1) los que afectan de manera universal, como el deterioro físico durante la vejez o la mortalidad, 2) los riesgos que afectan a grupos específicos de la población, como es el caso de las llamadas enfermedades profesionales, 3) los riesgos asociados al ciclo vital de las personas y 4) los riesgos intergeneracionales, ligados a la transmisión de rasgos adversos de padres a hijos, ya sea a nivel genético o sociocultural, ejemplo de lo anterior es la pobreza.

Se desprende una importante observación del concepto de vulnerabilidad social: la exposición al riesgo no implica necesariamente sucumbir ante la adversidad, sino que puede convertirse en una oportunidad de crecimiento, lo que nos acerca al concepto de resiliencia (Saavedra \& Castro, 2009). Lo anterior debe tenerse en cuenta a la hora de intervenir, ya que esos recursos se podrán usar como apoyo para hacer frente a la dificultad.

Otra precaución que hay que tener al acercarse al concepto de vulnerabilidad, se refiere a que al momento de medir dicho fenómeno, se dispondrá de múltiples métodos e instrumentos, tanto de carácter cuantitativo como cualitativo, ya que no existe un procedimiento único para dimensionar este elemento.

Medir el grado de vulnerabilidad de un determinado colegio o establecimiento educacional es algo que se viene realizando en Chile desde mediados 
de la década de 1990, por parte de la Junta Nacional de Auxilio Escolar y Becas (JUNAEB, 2010), para focalizar los recursos estatales destinados a velar por los niños, niñas y jóvenes chilenos en condición de vulnerabilidad biopsicosocial, para que ingresen, permanezcan y tengan éxito en el sistema educativo.

Hasta el año 2006, la JUNAEB proporcionaba un indicador denominado Índice de Vulnerabilidad Escolar (IVE), basado en una encuesta nacional dirigida principalmente a estudiantes de $1^{\circ}$ Básico y $1^{\circ}$ Medio de los establecimientos municipales y particulares subvencionados del país; a partir del año 2004, también se incluyeron los estudiantes que cursaban Prekinder o Kinder. A través de esta información, se calculaba el IVE del establecimiento, el cual indicaba el porcentaje estimado de la matrícula por nivel (Básica y Media) de cada establecimiento que se encontraba en condición de vulnerabilidad.

A partir del año 2007, se modifica el tipo de información recolectada por la encuesta nacional de la JUNAEB y se integran datos de los estudiantes y de sus familias, provenientes de diferentes instituciones: información socioeconómica de la familia de los estudiantes, así como el uso de las ayudas del Estado para superar la pobreza, información ofrecida por el Ministerio de Planificación (MIDEPLAN) a través de la Ficha de Protección Social y el Programa Chile Solidario; datos de protección social a niños y adolescentes vulnerados en sus derechos, datos ofrecidos por el Servicio Nacional del Menor (SENAME); la calidad de salud del estudiante y su familia según el Fondo Nacional de Salud (FONASA); datos de identificación de la familia (edad, número de hijos, estado civil, bienes, etc.) según el Registro Civil chileno y datos del Ministerio de Educación (MINEDUC), tales como matrícula y notas de los alumnos.

De esta manera se genera la base de datos del Registro Nacional de Información Social del Estudiante (RENISE) que sustenta al nuevo índice de vulnerabilidad denominado IVE-SINAE (Sistema Nacional de Asignación con Equidad), ponderado desde múltiples variables: salud, socioeconómica, sociodemográfica y uso de las redes de protección y reinserción social de niños y adolescentes (JUNAEB, 2005).

\section{Prácticas de enseñanza en sala de clase, cultura escolar e innovación}

Los estudios de la práctica de enseñanza en sala de clase están relacionados con la planificación (MINEDUC, 2003), la claridad de metas y clima emocional positivo que el profesor puede promover en el aula (Fernández \& Cuadrado, 2008; Unicef, 2004), las atribuciones y creencias que tiene de sí mismo y de sus estudiantes (Latorre, 2005; Torre \& Godoy, 2004), el dominio disciplinar y didáctico favorable al proceso de enseñanzaaprendizaje (Godino, Font, Wilhelmi \& De Castro, 2009), entre otros aspectos que operan como ejes organizadores de la acción docente.

La práctica de enseñanza no viene determinada por la formación inicial para docente (Cuadrado \& Fernández, 2008), sino que se construye en la experiencia de la docencia (Torre \& Godoy, 2004), cuya regularidad constituye la cultura escolar, es decir, un sistema de creencias compartido por la comunidad educativa, que se reconstruyen y transforman dinámicamente dentro de las opciones posibles que los códigos de dicho sistema cultural provee (Villalta, 2006).

Así, al caracterizar la práctica de enseñanza en sala de clase lo que se hace es dar cuenta de la regularidad que tiene la acción en el tiempo (Mares, Guevara, Rueda, Rivas \& Rocha, 2004; McFarland \& Bender-deMoll, 2004), y cómo esta integra los elementos que se introducen o emergen en la dinámica de sala de clase. En tal sentido, la innovación en la práctica docente en sala de clase es una actividad de mediación cultural de nuevos patrones al proceso de aprendizaje (Pasmanik \& Cerón, 2005). Los patrones de enseñanza se confirman o transforman en las interacciones dialogales en sala de clase (Cubero et al., 2008), y conocer su regularidad permite inferir los consensos subyacentes, y a veces explícitos, de profesor y alumnos sobre el cómo y el para qué educar en dicho contexto. 
Los establecimientos ubicados en contextos sociales vulnerables tienen altos índices de fracaso educativo, y entre las múltiples causas se encuentran la constitución de culturas escolares caracterizadas por el juicio compartido sobre las insuficientes capacidades cognitivas y de apoyo familiar de los alumnos y prácticas de enseñanza en sala de clase inefectivas de reproducción de contenidos, de control que no relacionan los diversos contenidos de enseñanza, ente otras (Román, 2003).

En contraparte, también se han estudiado los aspectos culturales y de enseñanza en sala de clase eficaces en el logro de aprendizajes (Jiménez, Riquelme, Vilos \& Ortiz, 2006; Laboratorio Latinoamericano de Evaluación de la Calidad de la Educación [LLECE], 2002; Murillo \& Román, 2009; Unicef, 2004). Sobre los elementos de la cultura escolar vinculados a logros de aprendizaje en alumnos de establecimientos educativos ubicados en sectores sociales vulnerables, destacan en directa relación con el clima social y actividades de enseñanza en el aula aspectos como: a) gestión pedagógica institucional orientada por proyecto educativo centrado en el aprendizaje de los alumnos, b) equipos directivos que lideran los procesos pedagógicos, c) reglas claras de funcionamiento para todos los miembros de la comunidad educativa, d) uso eficiente de los recursos disponibles y e) consolidación de una identidad institucional positiva.

Los estudios sobre práctica de enseñanza favorables a logros de aprendizaje de los estudiantes en contextos sociales vulnerables, describen acciones regulares o estables antes, durante y después del encuentro educativo de sala de clase, así como un juicio positivo de los alumnos e institución educativa. Se encuentra que las prácticas de enseñanza asociadas al aprendizaje de los alumnos comparten patrones de actividades que se pueden agrupar de la siguiente forma: a) trabajo en equipo por departamentos (MINEDUC, 2003, 2008; Unicef, 2004), b) trabajo docente personal y colectivo de reflexión de prácticas pedagógicas (Arroyave, 2008; Unidad de Gestión Pedagógica y Curricular [MINEDUC], 2008) y c) desarrollo de repertorio de estrategias de acción para promover aprendizajes (Fernández \& Cuadrado, 2008; LLECE, 2002).

\section{Metodología}

\section{Participantes}

Es un estudio cuantitativo de tipo descriptivo y correlacional. La población estuvo compuesta por alumnos y profesores de establecimientos de educación secundaria, administrados por el municipio y ubicados en sectores sociales vulnerables del país. La muestra es de tipo no probabilístico y polietápico, orientado por los objetivos del estudio. Se seleccionaron dos establecimientos de educación secundaria con alto logro educativo, en comparación con sus pares del mismo municipio, ubicados en sectores sociales vulnerables. Esta selección se realizó según el rango alcanzado por los alumnos en la Prueba Nacional de Medición de la Calidad Educativa (SIMCE, 2008) de Chile, que mide los aprendizajes en las asignaturas de Lengua Castellana y Matemáticas. En los establecimientos seleccionados, se encuestaron 502 alumnos de los dos primeros años de la educación secundaria y 39 profesores.

\section{Instrumentos}

Se aplicaron tres instrumentos:

\section{Cuestionario de Cultura Institucional}

Constituido por 16 ítems que indagan en la percepción que tienen alumnos y profesores de la gestión pedagógica de coordinar a los actores educativos hacia las metas institucionales. La validez de los ítems se realizó por criterio de 12 jueces, mediante la aplicación del coeficiente de Aiken, considerándose los ítems cuyo coeficiente de acuerdo está en el rango de 0.83 y 1.0 , es decir alto y muy alto nivel de acuerdo. El instrumento cuenta con muy alta confiabilidad (alfa de Cronbach $=0.85$ ). 


\section{Cuestionario sobre Trabajo}

Cotidiano de la Enseñanza

Instrumento de 15 ítems que indaga en juicios sobre las actividades de preparación, realización y evaluación de la interacción didáctica de sala de clase, así como el juicio sobre el rol de profesor y de los alumnos en el aula. El análisis de validez de los ítems se realizó por criterio de 12 jueces, aplicando el coeficiente de Aiken; el instrumento contiene ítems cuyo nivel de acuerdo entre los jueces está en el rango de 0.97 y 1.0, es decir muy alto acuerdo. El instrumento cuenta con alta confiabilidad (alfa de Cronbach $=0.72$ ).

\section{Escala de Resiliencia SV-RES}

(Saavedra Eु Villalta, 2008)

Instrumento de 60 ítems que indaga en dimensiones del entorno y de la historia personal vinculados a la resiliencia. Posee alta validez (correlación de Pearson $=0.75)$ evaluada a través del método interprueba con la escala CD-Risc (Connors \& Davison,
2002) y cuenta con una confiabilidad alta (alfa de Cronbach $=0.75$ )

\section{Resultados}

La presentación de los resultados sobre las variables de estudio se realiza en dos apartados: 1) alumnos y 2) profesores.

\begin{abstract}
Alumnos
La proporción de hombres y mujeres es similar en cada edad (Tabla 2) y la estimación de la confiabilidad a través de la consistencia interna y homogeneidad de los ítems en los tres instrumentos empleados va de alta a muy alta (Tabla 3).

Las mujeres tienen una mejor percepción de las Prácticas cotidianas de enseñanza en comparación con los hombres (Tabla 4). Aunque la puntuación promedio de las mujeres en la percepción de la Cultura Institucional y la Resiliencia es mayor que la de los hombres, estas diferencias no son estadísticamente significativas.
\end{abstract}

TABLA 2

Descripción de los casos (alumnos) por edad y sexo

\begin{tabular}{cccc}
\hline & \multicolumn{2}{c}{ Sexo } & Total(\%) \\
\cline { 2 - 3 } Edad & Hombre(\%) & Mujer(\%) & 22.5 \\
14 & 21.1 & 23.9 & 42 \\
15 & 41.4 & 42.6 & 24.3 \\
16 & 26.3 & 22.3 & 8.4 \\
17 & 9.2 & 7.6 & 2.6 \\
18 & 2 & 3.2 & 0.2 \\
19 & 0.0 & 0.4 & 100 \\
Total & 100 & 100 & $N=502$ \\
\hline
\end{tabular}

Fuente: elaboración propia.

TABLA 3

Fiabilidad de los instrumentos en alumnos

\begin{tabular}{lccc}
\hline \multicolumn{1}{c}{ Instrumentos } & № casos válidos & № ítems & alfa de Cronbach \\
\hline Cuestionario de Cultura Institucional. & 502 & 12 & 0.797 \\
Cuestionario de Prácticas cotidianas de enseñanza & 502 & 15 & 0.872 \\
Escala de Resiliencia SV-RES & 502 & 60 & 0.952 \\
\hline
\end{tabular}

Fuente: elaboración propia. 
TABLA 4

Comparación de media de instrumentos por género: Percepción del trabajo cotidiano de enseñanza, Cultura del Establecimiento y Resiliencia

\begin{tabular}{llcccccc}
\hline & Sexo & $N$ & Media & Desviación típ. & $\begin{array}{c}\text { Error típ. } \\
\text { de la media }\end{array}$ & \multirow{2}{*}{ Sig. (bilateral) } \\
\hline Cuestionario de Prácticas & Hombre & 251 & 58.13 & 10.185 & 0.643 & \multirow{2}{*}{2.069} & \multirow{2}{*}{0.039} \\
cotidianas de enseñanza & Mujer & 251 & 59.92 & 9.116 & 0.575 & & \\
Cuestionario de Cultura & Hombre & 251 & 46.52 & 7.414 & 0.468 & -1.33 & 0.184 \\
Institucional & Mujer & 251 & 47.36 & 6.805 & 0.43 & & \\
Escala de Resiliencia & Hombre & 251 & 257.51 & 24.574 & 1.551 & -1.778 & 0.076 \\
SV-RES & Mujer & 251 & 261.47 & 25.271 & 1.595 & & \\
\hline
\end{tabular}

Fuente: elaboración propia.

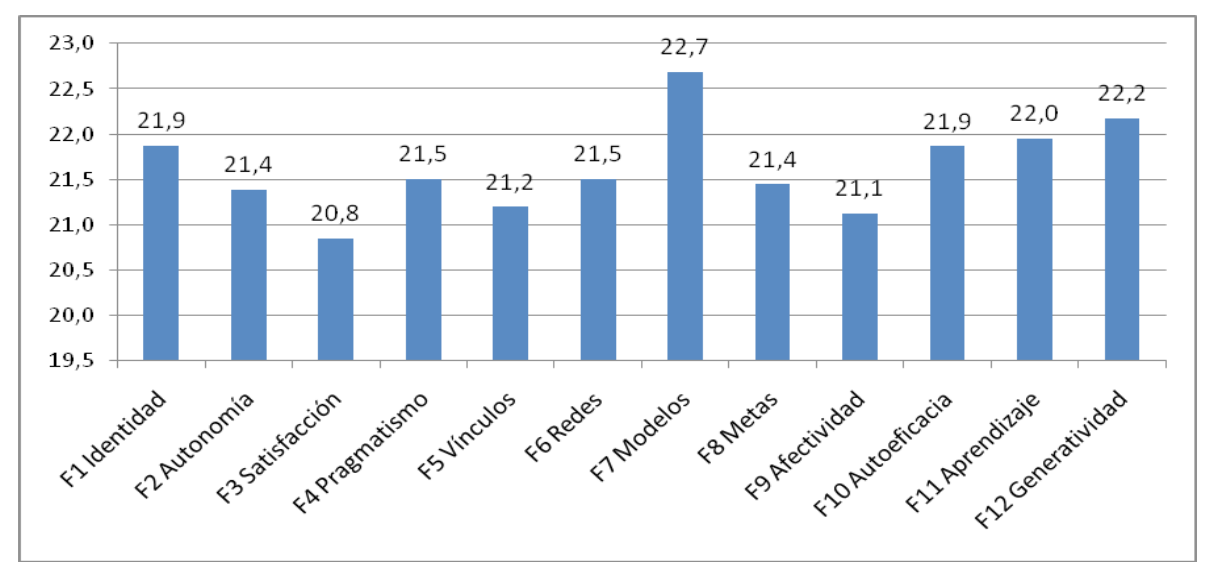

Figura 1. Puntajes promedio de los 502 casos de alumnos en las 12 dimensiones de Resiliencia de la Escala SV-RES. Fuente: elaboración propia.

TABLA 5

Correlación entre los tres instrumentos

\begin{tabular}{lll}
\hline & $\begin{array}{l}\text { Cuestionario de Prácticas } \\
\text { cotidianas de enseñanza. }\end{array}$ & $\begin{array}{l}\text { Cuestionario de Cultura } \\
\text { Institucional. }\end{array}$ \\
\hline Cuestionario de Prácticas cotidianas de enseñanza & 1 & $0.753(* *)$ \\
Cuestionario de Cultura Institucional & $0.753(* *)$ & 1 \\
Escala de Resiliencia SV-RES & $0.513(* *)$ & $0.440(* *)$ \\
\hline
\end{tabular}

$N=502, * * \mathrm{p}<0.01 ; * \mathrm{p}<0.05$

Fuente: elaboración propia.

El análisis de los resultados de todos los alumnos en el Cuestionario de Resiliencia (Figura 1), indica que la dimensión referida a Modelos -percepción de contar con personas cercanas para resolver situaciones problemas- y la dimensión Generatividad -valoración positiva de las propias capacidades para generar soluciones a sus problemas-, son las más altas. Mientras que las puntuaciones promedio bajan en la dimensión Satisfacción -agrado que tiene hacia sí mismo para abordar situaciones problemáticas-y la dimensión Afectividad -disposición para manifestar afectos positivos-. 
Es alta la asociación que hacen los alumnos entre la percepción de la Cultura Institucional y las Prácticas cotidianas de enseñanza (Tabla 5). Por otra parte, la correlación de ambos instrumentos con el cuestionario de Resiliencia es moderada.

\section{Profesores}

En el caso de los profesores, la proporción de hombres participantes del estudio es mayor que la de mujeres y más de la mitad tiene 41 años de edad o más (Tabla 6). Se encuentra que los tres instrumentos aplicados tienen muy alta confiabilidad de consistencia interna y homogeneidad de los ítems (Tabla 7).

No se encuentran diferencias significativas entre las respuestas de profesores y profesoras a los tres instrumentos del estudio (Tabla 8) y la correlación entre los cuestionarios de Cultura Institucional y Prácticas cotidianas de enseñanza es moderada; y

TABLA 6

Descripción de la muestra

\begin{tabular}{ccccc}
\hline & & \multicolumn{2}{c}{ Sexo } & \multirow{2}{*}{ Total } \\
\cline { 3 - 4 } & & Hombre(\%) & Mujer(\%) & \\
\hline \multirow{4}{*}{ Edad } & $26-30$ & 13 & 7 & 11 \\
& $31-35$ & 4 & 20 & 11 \\
& $36-40$ & 30 & 7 & 21 \\
\hline Total & más de 41 & 52 & 67 & 58 \\
\hline & & 100 & 100 & 100 \\
\hline
\end{tabular}

Fuente: elaboración propia.

TABLA 7

Análisis de la fiabilidad de los instrumentos

\begin{tabular}{lccc}
\hline & Casos válidos & № ítems & alfa de Cronbach \\
\hline Cuestionario de Cultura Institucional & 38 & 16 & 0.926 \\
Cuestionario de Prácticas cotidianas de la enseñanza & 37 & 15 & 0.890 \\
Escala de Resiliencia SV-RES & 34 & 60 & 0.963 \\
\hline
\end{tabular}

Fuente: elaboración propia.

TABLA 8

Comparación de medias de los tres instrumentos entre sexo

\begin{tabular}{llcccccc}
\hline & Sexo & $\begin{array}{c}\text { Casos } \\
\text { válidos }\end{array}$ & Media & $\begin{array}{c}\text { Desviación } \\
\text { est. }\end{array}$ & $\begin{array}{c}\text { Error típ. } \\
\text { de la media }\end{array}$ & $t$ & Sig. (bilateral) \\
\hline Cuestionario de Cultura & Hombre & 23 & 62.26 & 9.635 & 2.009 & 0.045 & 0.965 \\
Institucional & Mujer & 16 & 62.06 & 17.957 & 4.489 & & \\
Cuestionario de Prácticas & Hombre & 23 & 60.61 & 15.183 & 3.166 & -0.289 & 0.774 \\
cotidianas de la enseñanza & Mujer & 16 & 62.13 & 17.427 & 4.357 & & \\
Escala de Resiliencia SV- & Hombre & 23 & 275.65 & 21.187 & 4.418 & -1.693 & 0.099 \\
RES & Mujer & 16 & 285.94 & 14.177 & 3.544 & & \\
\hline
\end{tabular}

Fuente: elaboración propia. 
TABLA 9

Correlación entre instrumentos: Cultura Institucional, Prácticas cotidianas de enseñanza y Resiliencia

\begin{tabular}{lcc}
\hline & $\begin{array}{c}\text { Cuestionario de Prácticas } \\
\text { cotidianas de la enseñanza }\end{array}$ & $\begin{array}{c}\text { Cuestionario de Cultura } \\
\text { Institucional }\end{array}$ \\
\hline Cuestionario de Prácticas cotidianas de la enseñanza & 1 & $0.588(* *)$ \\
Cuestionario de Cultura Institucional & $0.588(* *)$ & 1 \\
Escala de Resiliencia SV-RES & -0.031 & 0.052 \\
\hline
\end{tabular}

$N=37 ; * \mathrm{p}<0.01 ; \mathrm{p}<0.05$

Fuente: elaboración propia.

TABLA 10

Ítems de los cuestionarios Cultura Escolar y Prácticas cotidianas de enseñanza que tienen alta correlación de Pearson

\begin{tabular}{|c|c|c|c|}
\hline $\begin{array}{r}\text { Ítems de cuestionario de Prácticas de } \\
\text { enseñanza }\end{array}$ & $\begin{array}{l}\text { 19. Planifico mi activi- } \\
\text { dad educativa de for- } \\
\text { ma coordinada con el } \\
\text { resto del profesorado } \\
\text { (ya sea por nivel, ciclo, } \\
\text { departamentos). }\end{array}$ & $\begin{array}{l}\text { 24. Considero que } \\
\text { facilito la adquisición } \\
\text { de nuevos contenidos } \\
\text { a través de las estrate- } \\
\text { gias que utilizo. }\end{array}$ & $\begin{array}{l}\text { 25. Propongo a mis } \\
\text { estudiantes activida- } \\
\text { des variadas de apren- } \\
\text { dizaje. }\end{array}$ \\
\hline $\begin{array}{l}\text { 1. Creo que la meta de directivos, alumnos, } \\
\text { profesores y apoderados del establecimiento } \\
\text { está centrada en el aprendizaje. }\end{array}$ & $0.579(* *)$ & $0.641(* *)$ & 00.264 \\
\hline $\begin{array}{l}\text { 3. Considero que el equipo directivo } \\
\text { lidera los procesos de mejoramiento de } \\
\text { aprendizajes de los estudiantes. }\end{array}$ & $0.654(* *)$ & 00.276 & $0.459(* *)$ \\
\hline $\begin{array}{l}\text { 4. Considero que en el establecimiento } \\
\text { existen reglas claras y explícitas para los } \\
\text { alumnos. }\end{array}$ & $0.673(* *)$ & $0.449(* *)$ & $0.481(* *)$ \\
\hline $\begin{array}{l}\text { 8. Considero que en el establecimiento se } \\
\text { aprovechan adecuadamente los recursos } \\
\text { humanos y materiales disponibles. }\end{array}$ & $0.602(* *)$ & $0.497(* *)$ & $0.515(* *)$ \\
\hline $\begin{array}{l}\text { 13. Considero que en este establecimiento } \\
\text { funciona correctamente el consejo de } \\
\text { profesores. }\end{array}$ & $0.641(* *)$ & $0.441(* *)$ & $0.639(* *)$ \\
\hline
\end{tabular}

$N=37 ; * p<0.01 ; * p<0.05$

Fuente: elaboración propia.

es nula la correlación entre los cuestionarios y la puntuación de la escala de Resiliencia (Tabla 9).

La correlación entre los ítems del cuestionario de Cultura Institucional y el cuestionario de las Prácticas Cotidianas de la enseñanza, ponen en evidencia alta correlación entre algunos ítems (Tabla 10).

\section{Discusión y conclusiones}

Para los alumnos, las percepciones de las prácticas de enseñanza y de la cultura institucional escolar están relacionadas. La valoración positiva que tienen los alumnos de sus profesores es lo que probablemente se transfiere a todo el funcionamiento del 
establecimiento, con incidencia en los resultados de aprendizaje. Como ha sido descrito en otros estudios (LLECE, 2002; Unicef, 2004), esta dinámica virtuosa está alimentada por equipos directivos que coordinan las acciones hacia metas educativas y por docentes con competencias comunicativas, para crear climas afectivos favorables al aprendizaje (Fernandez \& Cuadrado, 2008).

Las mujeres tienen un mejor aprecio de la experiencia de sala de clase en comparación con los varones. Es probable que ellas sean más empáticas con los esfuerzos de enseñanza de sus profesores y aprovechen de forma más constante la experiencia educativa que les ofrece el establecimiento.

Respecto del perfil de resiliencia de los estudiantes, este refleja una gran capacidad para seguir modelos, generar respuestas alternativas frente a los problemas y aprender de las experiencias positivas y negativas. Lo anterior confirma otros estudios sobre resiliencia en estudiantes secundarios de sectores vulnerables (Saavedra \& Yanquez, 2008; Villalta, 2009) y además indica un terreno fértil para que los docentes puedan fortalecer y/o promover patrones innovadores de interacción didáctica en la sala de clase.

Las áreas de resiliencia disminuidas en los estudiantes como la satisfacción, la afectividad y los vínculos, están en directa relación con la etapa de desarrollo que atraviesan los alumnos adolescentes, caracterizada por vínculos afectivos más inestables, procesos identitarios cargados de inconformismo hacia el sistema adulto, que los lleva a tener sensaciones de insatisfacción con lo que viven. Probablemente, esto añada particulares desafíos a la creación de climas favorables al aprendizaje en sectores sociales vulnerables, que son posibles de superar desde la perspectiva de las escuelas eficaces (Unicef, 2004).

Aunque la correlación es moderada, se encuentra que la experiencia escolar tiene relación con la resiliencia. Para los adolescentes de sectores sociales vulnerables la experiencia escolar es un recurso de protección y también de promoción humana. Asimismo, el resultado da cuenta de cómo la experiencia escolar está incrustada en la experiencia de esfuerzo y realización que marca la percepción de resiliencia del adolescente y que, posiblemente, proyecta a otros aspectos de su vida.

En los profesores de establecimientos ubicados en sectores sociales vulnerables, los resultados indican que, en general, los juicios de sus prácticas de enseñanza son independientes de los juicios que tienen de la institución escolar en la cual trabajan. De modo específico, se encuentra asociación entre juicios sobre aspectos referidos a la claridad de metas institucionales y planificación de la enseñanza. Como lo reportan algunos estudios (Murillo \& Román, 2009; Unicef, 2004), el trabajo en equipo y el liderazgo de los directivos son valorados en relación con la planificación e implementación de diversas estrategias de enseñanza en el aula, que aseguran prácticas de enseñanza eficaces y pertenencia institucional.

En el caso de los profesores, hay nula relación entre el juicio que tengan de sus prácticas de enseñanza y de la cultura institucional con la resiliencia. Es probable que la resiliencia de los profesores se alimente o sostenga en otros espacios y redes sociales. Es muy probable también que los profesores tengan mayor capital cultural que sus alumnos de sectores sociales vulnerables, lo que explica el por qué para unos la resiliencia está más vinculada a la experiencia escolar -los alumnos- que para los otros -los profesores-. Queda por profundizar en futuros estudios cuáles aspectos de la vida escolar son particularmente significativos para los alumnos y profesores, y cómo estos se construyen en las interacciones cotidianas de la vida escolar, especialmente en la sala de clase, donde ambos interactúan la mayor parte del tiempo escolar.

\section{Referencias}

Arroyave, D. (2008). Investigar: una estrategia de actuación para el rol del docente actual. Revista Investigaciones en Educación, 8(1), 63-81.

Chambers, R. (1989). Editorial Introduction: Vulnerability, coping and policy [Monográfico: Vulnerability: How the Poor Cope]. IDS Bulletin, 20(2), 1-7.

Chile, Ministerio de Educación, Unidad de Gestión Pedagógica y Curricular. (2008). Planificación y diseño de la enseñanza. Santiago: Autor. 
Chile, Ministerio de Educación. (2003). Marco para la Buena Enseñanza. Santiago: Autor.

Chile, Junta Nacional de Auxilio Escolar y Becas \& Sistema Nacional de Asignación con Equidad para Becas. (2005). Una nueva visión en la construcción de igualdad de oportunidades en la infancia [Versión electrónica]. Disponible en http://www.junaeb.cl

Chile, Junta Nacional de Auxilio Escolar y Becas. (2010, julio). Los requerimientos de JUNAEB a la Ficha de Protección Social (FPS). Noticias. Extraído el 20 de julio, 2010, de http://www.junaeb.cl/prontus_junaeb/site/artic/20100714/pags/20100714093158. html

Cuadrado, I. \& Fernández, I. (2008). iCómo intervienen maestros y profesores para favorecer el aprendizaje en secundaria? Un estudio comparativo desde el análisis del discurso. Infancia y Aprendizaje, 31(1), 3-23.

Cubero, R., Cubero, M., Santamaría, A., De La Mata, M., Ignacio, C. \& Prados, M. (2008). La educación a través de su discurso. Prácticas educativas y construcción discursiva del conocimiento en el aula. Revista de Educación, 346, 71-104.

Esping-Andersen, G. (2000, mayo). Social indicators and welfare monitoring. Programme Paper Number 2, Social Policy and Development, Ginebra Instituto de Investigaciones de las Naciones Unidas para el Desarrollo Social (UNRISD).

Fernández, I. \& Cuadrado, I. (2008). iSon conscientes los profesores de secundaria de los recursos comunicativos verbales y no verbales que emplean en el aula? Revista Iberoamericana, 46, 1-13.

Godino, J., Font, V., Wilhelmi, M. \& De Castro, C. (2009). Aproximación a la dimensión normativa en didáctica de las Matemáticas desde un enfoque ontosemiótico. Enseñanza de las Ciencias, 27(1), 59-76.

Goodyer, I. (1995). Risk and resilience processes in childhood and adolescent. London: Social Pediatrics.

Grotberg, E. (1995). A guide to promoting resilience in children. The Bernard van Leer Foundation, The Hague [En línea]. Recuperado el 20 de marzo de 2008, de http://resilnet.uiuc.edu

Grotberg, E. (1999). The International Resilience Project. En R. Roth (Ed.), Psychologists facing the chal- lenge of global culture with human rights and mental health (pp. 239-256). Lengerich, Germany: Pabst. Guidano, V. (1994). El sí mismo en proceso. Madrid: Paidós.

Jiménez, J., Riquelme, G., Vilos, V. \& Ortiz, H. (2006). La calidad educativa asociada a la gestión de la escuela [Versión electrónica]. Revista Vasconcelos de Educación, 2(3), 110-126. Recuperado el 20 de abril de 2009, de http://www.itson.mx/vasconcelos/ documentos/volII-num3/RVE-2-3-10.pdf

Kreisler, L. (1996). La résilience mise en spirale. Spirale, $1,162-165$.

Laboratorio Latinoamericano de Evaluación de la Calidad de la Educación. (2002). Estudio cualitativo de escuelas con resultados destacables en siete países latinoamericanos. Santiago: UNESCO, Oficina Regional de Educación para América Latina y el Caribe.

Latorre, M. (2005, 10 de junio). Continuidades y rupturas entre formación inicial y ejercicio profesional docente. Revista Iberoamericana de Educación, 36(2), 1-12. Recuperado el 15 de enero de 2010, de http://dialnet.unirioja.es/servlet/

dcart info $=$ link $\&$ codigo $=3130480$ \&orden $=235695$

Lutha, S. \& Cicchetti, D. (2000). The construct of resilience: Implications for interventions and social policy. Development and Psychopathology, 12(4), 857-885.

Manciaux, M. (2005). La resiliencia: resistir y rehacerse. Madrid: Gedisa.

Mares, G., Guevara, Y., Rueda, E., Rivas, O. \& Rocha, H. (2004). Análisis de las interacciones maestraalumnos durante la enseñanza de las ciencias naturales en primaria. Revista Mexicana de Investigación Educativa, 9(22), 721-745.

Masten, A. (1990). Resilience and development. Development and Psychopathology, 2(4), 425-444.

McFarland, D. A. \& Bender-deMoll, S. (2004, agosto). Classroom structuration: A study of network stabilization. Paper presented at the annual meeting of the American Sociological Association, San Francisco, CA, United States.

Moser, C. (1998). The asset vulnerability framework: Reassessing urban poverty reduction strategies. World Development, 26(1), 1-19. 
Murillo, J. \& Román, M. (2009). Mejorar el desempeño de los estudiantes de América Latina. Algunas reflexiones a partir de los resultados del SERCE. Revista Mexicana de Investigación Educativa, 14, 451-484.

Pasmanik, D. \& Cerón, R. (2005). Las practicas pedagógicas en el aula como punto de partida para el análisis del proceso enseñanza-aprendizaje: un estudio de caso en la asignatura de química. Estudios Pedagógicos, 31(2), 71-87.

Perona, N. \& Rocchi, G. (2001). Vulnerabilidad y exclusión social. Una propuesta metodológica para el estudio de las condiciones de vida de los hogares. Revista KAIROS, 8. Extraído el 15 mayo, 2010, de http://www2.fices.unsl.edu.ar/ kairos/k08-08. htm\#arriba

Román, M. (2003). ¿Por qué los docentes no pueden desarrollar procesos de enseñanza- aprendizaje de calidad en contextos sociales vulnerables? Persona y Sociedad, 17(1), 113-128.

Rutter, M. (1993). Resilience: Some conceptual considerations. Journal of Adolescent Health, 14(8), 626-631.

Saavedra, E. (2003). El Enfoque Cognitivo Procesal Sistémico, como posibilidad de intervenir educativamente en la formación de sujetos resilientes: estudio de casos. Tesis Doctoral, Universidad de Valladolid, España. Saavedra, E. (2005). Resiliencia: la historia de Ana y Luis. Liberabit, 11, 91-101.

Saavedra, E. \& Villalta, M. (2008a). Escala de Resiliencia SV-RES, para jóvenes y adultos. Santiago: CEANIM.
Saavedra, E. \& Villalta, M. (2008b). Estudio comparativo en resiliencia con sujetos discapacitados motores. Revista Investigaciones en Educación, 8(1), 81-90.

Saavedra, E. \& Yanquez, E. (2008, octubre). Jóvenes infractores de ley, resiliencia y educación. Ponencia presentada en las VIII Jornadas Internacionales de Psicología, Lima, Perú.

Saavedra, E. \& Castro, A. (2009). Escala de Resiliencia Escolar, ERE. Santiago: CEANIM.

Torre, C. \& Godoy, A. (2004). Diferencias individuales en las atribuciones causales de los docentes y su influencia en el componente afectivo. Revista Interamericana de Psicología, 38(2), 217-224.

Fondo de Naciones Unidas para la Infancia. (2004). ¿Quién dijo que no se puede? Escuelas efectivas en sectores de pobreza. Santiago de Chile: Ministerio de Educación/Unicef. Recuperado el 20 de mayo de 2009, de http://scholar.google.com/scholar?hl $=$ en\&btnG $=$ Search\&q=intitle: $: Q u i e ́ n+$ dijo $+q$ ue + no + se + puede?+Escuelas +efectivas + en + se ctores + de + pobreza\#0

Vanistendael, S. (2002). La felicidad es posible. Madrid: Gedisa.

Villalta, M. (2006). Construcción de la práctica pedagógica del docente en la sala de clase. Algunos elementos para la innovación. Revista Investigaciones en Educación, 6(1), 59-89.

Villalta, M. (2009). Factores de resiliencia en estudiantes secundarios. Una propuesta de medición. Santiago: Universidad del Desarrollo. 